\title{
A Fresnelet-Based Encryption of Medical Images using Arnold Transform
}

\author{
Muhammad Nazeer ${ }^{1}$, Bibi Nargis ${ }^{2}$, Yasir Mehmood Malik ${ }^{3}$, and Dai-Gyoung Kim ${ }^{1 *}$ \\ ${ }^{1}$ Department of Applied Mathematics, Hanyang University, Ansan, 426-791, South Korea \\ ${ }^{2}$ School of Computer Science, University of Manchester, Manchester, UK \\ ${ }^{3}$ Department of Neurology, Rashid Hospital, Dubai, UAE
}

\begin{abstract}
Medical images are commonly stored in digital media and transmitted via Internet for certain uses. If a medical information image alters, this can lead to a wrong diagnosis which may create a serious health problem. Moreover, medical images in digital form can easily be modified by wiping off or adding small pieces of information intentionally for certain illegal purposes. Hence, the reliability of medical images is an important criterion in a hospital information system. In this paper, the Fresnelet transform is employed along with appropriate handling of the Arnold transform and the discrete cosine transform to provide secure distribution of medical images. This method presents a new data hiding system in which steganography and cryptography are used to prevent unauthorized data access. The experimental results exhibit high imperceptibility for embedded images and significant encryption of information images.
\end{abstract}

Keywords-Fresnelet transform; wavelet transform; Arnold transform, data hiding; and image encryption

\section{INTRODUCTION}

Due to recent expansions of information technology, the circulation of medical images among hospitals has become a common trend. Medical images are often distributed for specific purposes such as for teleconferences and interdisciplinary exchanges among medical personnel [1]. The transmission of medical imaging should be carefully treated to ensure reliability and authenticity verification [2]. Privacy is also a critical issue in medical images especially in case of medical jurisprudence where a small manipulation of brain contusion or hairline bony fractures can alter the situation drastically. For interdisciplinary data exchange clarity of image is mandatory such as in multiple sclerosis, a disease of nervous system [3]. If small demyelinating plaques are missed in this disease, whole the diagnostic picture, treatment plan, and even prognostic outcome would be altered. Similarly in infectious diseases of chest and abdomen or minimal trauma, if any misinterpretation is found because of imaging defect, clinical outcome would be seriously affected. Thus it is essential to ensure the protection of image information for both legislative and diagnostic reasons. A digital data hiding technique can provide this needed protection by embedding medical information data into other data (called the host or cover data) and has been developed for information security that is strongly based on cryptography and steganography [4].

Steganography and cryptography are both used for data confidentiality. Cryptography is employed for scrambling meaningful information into uncorrelated data keeping the contents of a message secret, whereas steganography hides the significant information under some cover data keeping the existence of a message secret [5]. Each technique enhances the security and privacy for protection of information data. Furthermore, combining both schemes into one system is likely to provide even better security and confidentiality [6]. In other words, steganography prevents an unintended recipient from suspecting the existence of data and the security of the steganography system relies on secrecy of the data encoding system $[7,8]$.

One way of data hiding entails the manipulation of the least significant bit (LSB) plane, from direct replacement of the cover LSB's with message bits to some type of logical or arithmetic combination between the cover image and the amount of information data which needs to be hidden. Several examples of LSB schemes have been presented in [9-11]. Mainly this technique achieves both high capacity and low perceptibility. However, this embedding scheme only overwrites the LSB plane of the cover image with the secret bit stream according to a pseudorandom number generator (PRNG). As a result, some structural asymmetry (never decreasing even pixels and increasing odd pixels when hiding the data) is introduced so that LSB makes the disclosure of hidden message very easy even at a low embedding rate using some reported steganalytic algorithms, such as the Chisquared attack, regular/singular groups (RS) analysis, sample pair analysis [10], and the general framework for structural steganalysis [12].

Most existing steganographic approaches usually assume that the LSB of natural cover images is insignificant and random enough, and thus those pixels/pixel pairs for data hiding can be selected freely using a PRNG. However, such an assumption is not always true, especially for images with many smooth regions [10]. Based on the stated researches, we found that natural images usually contain some flat regions as small as 5 by 5 blocks which are hard to be noticed. The LSB's in those regions have the values 1 or 0 . Therefore, embedding the secret data into these regions will make the LSB of stegano-images more and more random, which may lead to visual and statistical differences between cover and stegano images appearing as a noise-like distribution. The pixel-value differencing (PVD)-based scheme [13] is another kind of edge adaptive scheme which determines the number of embedded bits by the difference between a pixel and its neighbor. This shows the larger the difference is, the larger the

This work was supported by the National Research Foundation of Korea (NRF) grant funded by the Korea government (MEST) (NRF-2011-0026245). 
number of secret bits can be embedded. Based on our explorative experiments, however, we found that the existing PVD-based approaches cannot make full use of edge information for data hiding, so that the embedded image data does not have a good perceptibility.

Chang et al. extended Iwata et al.'s idea [14-16] and presented a lossless steganographic scheme for hiding secret data in each block of quantized DCT coefficients in JPEG images [17]. This method uses two successive zero coefficients of the medium-frequency components in each block to hide secret data. They further modified the quantization table to maintain the quality of the stegano-image while concealing a higher payload compared with Iwata et al.'s method. Their scheme achieved reversibility and acceptable image quality of the stegano-image at the same time. However, this scheme can only embed secret bits into the zero coefficients located in the successive zero coefficients in the medium area; non-zero coefficients in the medium area cannot be used. Later, Lin et al. embedded the secret values into the middle frequency of the quantized DCT coefficients, and reduced nonzero values of the quantized DCT coefficients which participate in the data hiding procedure. The aim was to design an adaptive and reversible DCT-based data hiding scheme [18]. Lin and Shiu combined Chang et al.'s [17] method and designed a 2-layer data hiding scheme for DCTbased images. This scheme outperforms Chang et al.'s scheme [16] in hiding capacity but the size of the hidden secret data is still less than $70 \mathrm{k}$ bits on average because it retains the reversibility function.

A medical image is distributed among a number of clinicians in telediagnosis and teleconsultation which require maximum information exchange over an unsecure network. Disclosing the information about an important patient's medical condition to general public can be a confidentiality risk. Access to medical information especially image data during transmission which should not be granted to an unauthorized party, demands an important confidentiality measurement. The integrity, on the other hand, demands that images should not be modified in the processes of transmission [19]. However, emerging technologies in field of medical imaging lead to the high demand of protection and confidentiality of the medical information, which must follow strict ethics and legislatives rules. Once the medical images are stolen or abused, the rights of the patients will suffer violation. Tso, H.-K, et al [20] proposes a secret sharing scheme to protect the security of the medical images based on the firstly secret sharing technique proposed by Shamir [21]. According to this technique, no one can obtain any information from one of the shared images unless acquiring the hidden information from all the authorized users. Furthermore, this technique is not efficient for embedding the complete information of secret data in one cover image [22]. Conventionally, information sharing and data hiding are two irrelevant concerns in the field of secure distribution of the information data. In our proposed method, however, a complete information data (e.g., medical image) can be embedded in single cover image (such as patient identity image or face image), rather than distributing in several cover images.
In this study, we proposed a novel technique for data hiding based upon the Fresnelet transform [23] along with applications of discrete cosine transform (DCT) and the Arnold transform [24]. The method based on the Fresnelet transform was initially designed for reconstruction of high resolution digital holography. Irrespective of traditional optical holography, the digital holography has a significant advantage of fast reconstruction process for target object data [25]. Therefore, the Fresnelet transform can be applied to protecting copyrights of digital multimedia contents and data hiding [26]. Besides it is recommended to make use of the Fresnelet transform to complex encryption procedures for providing more security and reliability to information data. Using the Fresnelet transform, an extracted information image data from embedded (cover) image can be obtained with high resolution [27]. In this regard, our proposed method will be useful for data hiding of medical data in the rapid digital communication of medical imaging system. Preserving the high resolution of medical image data at embedding stage and retrieving the precise resolution at extraction stage are the keys to more precise understanding of the anatomy. The precise retrieve of medical data can support early detection of abnormalities and increase the accuracy in the assessment of the size and morphology of the organs, effectively [28].

In this paper, we propose a data hiding algorithm to improve security and privacy by integrating both steganography and cryptography with an efficient embedding and extracting process of large size information data. Note that in the proposed method, the Fresnelet transform is employed in order to build a more flexible data hiding system than one using only the Fresnel transform [29] or the wavelet transform [30]. One of the main features of the proposed method is a multi-scale distribution of information on the Fresnelet transform domain yielding robust key parameters for security and privacy. In our presented method, it is not possible to attain the hidden information data without the precise key parameters, even if an attacker perceives the embedding algorithm of the method.

This paper is organized as follows. In Section II, the Fresnelet transform and Arnold transform are reviewed as the basic transforms in the proposed algorithm. The embedding and extraction schemes based on these basic transforms are presented in Section III. Numerical simulations and analysis are conducted to evaluate the proposed method in Section IV. In Section V, conclusions are discussed.

\section{BASIC TRANSFORMS}

\section{A. Fresnelet transform}

A new class of multiresolution bases can be obtained by applying the Fresnel transform to a wavelet basis. The Fresnelet transform has been used for image reconstructions from digital holograms with various parameters related to wavelength, resolution scale, and distance between object and image plane. A review of the Fresnel transform follows from [23].

The Fresnel transform is used to approximately model diffraction phenomena through the propagation of complex 
waves [29]. The one-dimensional Fresnel transform is defined on a function $f \in L_{2}(\mathbb{R})$ as the convolution integral:

$$
\tilde{f}_{\tau}(x)=\left(f * k_{\tau}\right)(x) \text { with } k_{\tau}(x)=\frac{1}{\tau} \exp \left(i \pi \frac{x^{2}}{\tau^{2}}\right)
$$

where $\tau>0$ is the parameter related to wavelength and the distance at a diffracted wave. The two-dimensional Fresnel transform is obtained by convolving with the tensor product of the one-dimensional kernel $k_{\tau}(x)$. That is, for $f \in L_{2}\left(\mathbb{R}^{2}\right)$,

$$
\tilde{f}_{\tau}(x, y)=\left(f * K_{\tau}\right)(x, y) \text { with } K_{\tau}(x, y)=k_{\tau}(x) k_{\tau}(y)
$$

Notice that the kernel $K_{\tau}(x, y)$ is separable, so that the useful properties of one-dimensional Fresnel transform are readily extended to two-dimensions. The Fresnel transform has many useful properties. One is the unitary property: processing via the Fresnel transform provides a perfect reconstruction of given data.

The two-dimensional wavelet is obtained from a onedimensional wavelet by separable extension. The wavelet transform is also defined on $L_{2}(\mathbb{R})$ as convolution integrals with a two parameter family $\left\{\psi_{j, l}\right\}_{j, l \in \mathbb{Z}}$, which forms a Riesz basis for $L_{2}(\mathbb{R})$, where

$$
\left\{\psi_{j, l}(x)=2^{j / 2} \psi\left(2^{j} x-l\right)\right\}_{j, l \in \mathbb{Z}} .
$$

The simplest example of a wavelet is the Haar wavelet that generates an orthonormal basis for $L_{2}(\mathbb{R})$. Processing via the wavelet transform provides a perfect reconstruction and a multiresolution decomposition of the data, as well. The properties of wavelet transforms are given in [14]. By applying the Fresnel transform to the wavelet basis, the Fresnelet basis is defined as follows:

$$
\left\{\left(\psi_{j, l}\right)_{\tau}^{\sim}\right\}_{j, l \in \mathbb{Z}} \text { with }\left(\psi_{j, l}\right)_{\tau}^{\sim}(x)=2^{j / 2} \tilde{\psi}_{2^{j}}\left(2^{j} x-l\right)
$$

With an orthogonal wavelet basis $\left\{\psi_{j, l}\right\}_{j, l \in \mathbb{Z}}$, we can have an orthonormal Fresnelet basis. For fixed $\tau$, by letting $\theta_{j, l}(x)=\left(\psi_{j, l}\right)_{\tau}^{\sim}(x)$, we have a Fresnelet decomposition:

$$
f=\sum_{j, l} c_{j, l} \theta_{j, l} \text { with } c_{j, l}=\left\langle f, \theta_{j, l}\right\rangle .
$$

Here, the coefficients $c_{j, l}$ are called the Fresnelet coefficients. From the separable nature of the Fresnelet, we may extend the one-dimensional Fresnelet transform to twodimensional space. In this case, four combinations of tensor products are obtainable:

$$
\begin{aligned}
& \Theta_{L L}=\left(\phi_{j, l}\right)_{\tau}^{\sim}(x)\left(\phi_{j, l}\right)_{\tau}^{\sim}(y) \\
& \Theta_{L H}=\left(\phi_{j, l}\right)_{\tau}^{\sim}(x)\left(\psi_{j, l}\right)_{\tau}^{\sim}(y) \\
& \Theta_{H L}=\left(\psi_{j, l}\right)_{\tau}^{\sim}(x)\left(\phi_{j, l}\right)_{\tau}^{\sim}(y)
\end{aligned}
$$

$$
\Theta_{H H}=\left(\psi_{j, l}\right)_{\tau}^{\sim}(x)\left(\psi_{j, l}\right)_{\tau}^{\sim}(y)
$$

where $\phi$ and $\psi$ are the scaling function and wavelet function, respectively, generating low-pass and high-pass filters, respectively. Applying the basis function above to data $f$, we obtain the four types of Fresnelet coefficients:

$$
\begin{aligned}
& C_{L L}=\left\langle f, \Theta_{L L}\right\rangle, C_{L H}=\left\langle f, \Theta_{L H}\right\rangle, \\
& C_{H L}=\left\langle f, \Theta_{H L}\right\rangle, C_{H H}=\left\langle f, \Theta_{H L}\right\rangle .
\end{aligned}
$$

The coefficient $C_{L L}$ is the low-passed data and the others are the high-passed data. Fig. 2 shows the Fresnelet coefficients of (6) applied to the medical information data shown in Fig. 1. In Fig. 2, note that the information image data is encrypted by the Fresnelet transform.

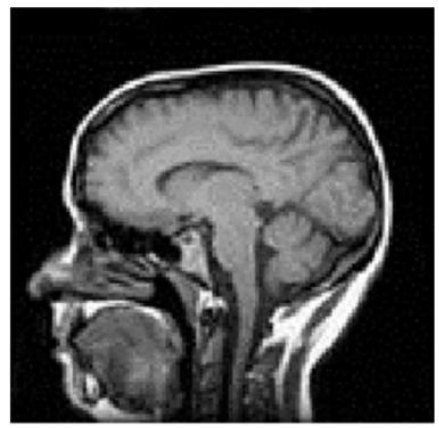

Fig.1. Medical image for data hiding.

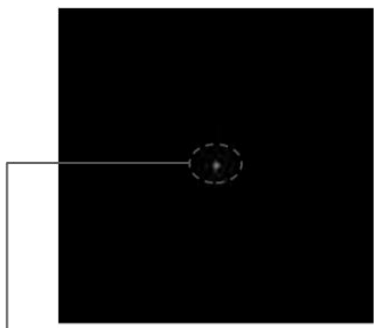

(a)

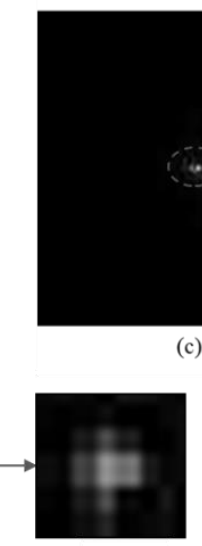

(e)

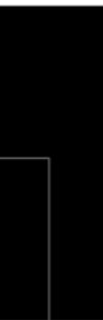

(c)

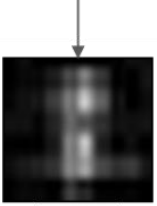

(f)

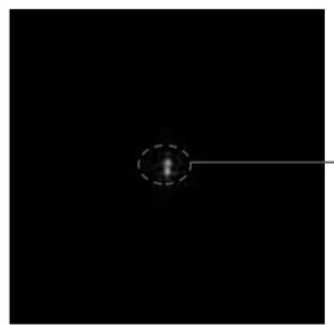

(b)

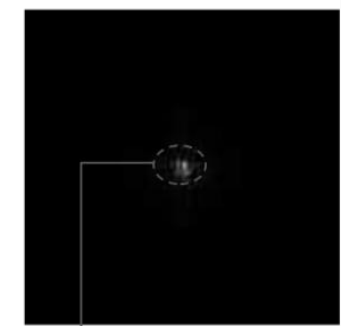

(d)

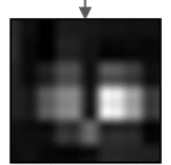

(g)

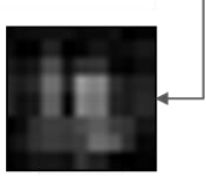

(h)
Fig.2. The magnitude of complex data sets of the Fresnelet coefficients applied to the medical information data shown in Fig. 1: (a) approximate data, (b) horizontal detail data, (c) vertical detail data, (d) diagonal detail data. Moreover, the (e), (f), (g), and (h) are the zooming parts of marked portion of (a), (b), (c), and (d). 
Thanks to the unitary property of the Fresnel transform and wavelet transform, data reconstruction is readily performed by applying the transpose of the forward processing as the inverse Fresnelet transform. Fig. 3 shows the inverse transformed data from Fig. 2, and Fig. 4 shows the result of the reconstruction of the medical information data from the data in Fig. 3. Note here that the proposed transform algorithm depends on the distance and the wavelength key parameters, which are very important for exact reconstruction of the information data. Furthermore, the nature of the reconstruction image is a complex field due to the propagation of complex waves. The complex image is beneficial for medical analysis based on multiresolution wavelet bases, just as for applications to digital holography [23, 25-27].

For the Fresnelet transform application to the data shown in Fig. 2, the encrypted information data may be covered to protect against a high degree of scrutiny. Also, in the reconstruction phase, the complete information may be acquired by using the exact keys as shown in Fig. 4..

\section{B. Arnold transform}

It is known that the Arnold transform works well in applications for encrypting images [5]. For an $N \times N$ image, the Arnold transform, for example, is given as

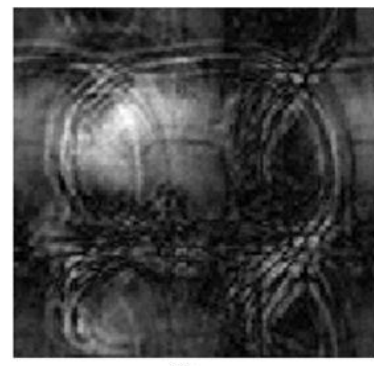

(a)

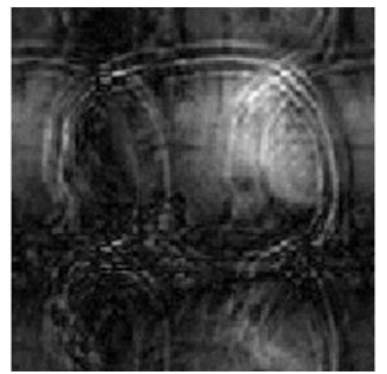

(c)

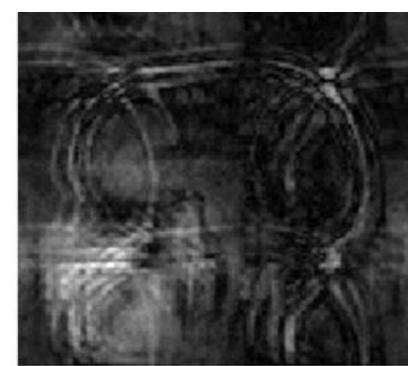

(b)

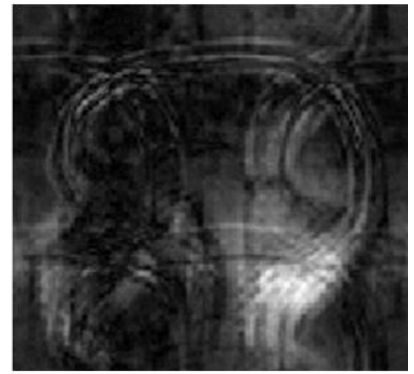

(d)
Fig.3. The magnitude of the inverse Fresnelet transformed data from the four sub-bands of complex data shown in Fig. 2: (a) from approximate data, (b) from horizontal detail data, (c) from vertical detail data, (d) from diagonal detail data.

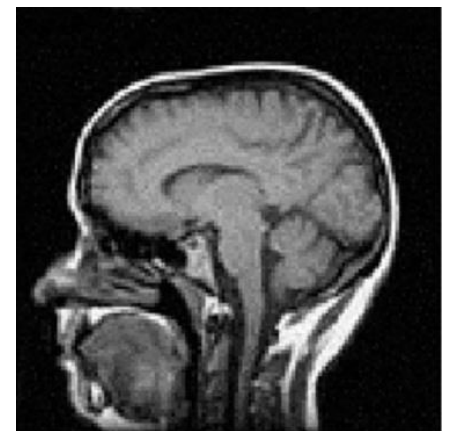

Fig.4. The reconstruction of the medical information data by combining with the magnitude of four sub-bands of complex data shown in Fig. 3.

$$
\left[\begin{array}{l}
x \\
y
\end{array}\right]=\left[\begin{array}{ll}
1 & 1 \\
1 & 2
\end{array}\right]\left[\begin{array}{l}
a \\
b
\end{array}\right](\bmod N)
$$

where $(a, b)$ and $(x, y)$ express the pixel co-ordinates of the original and encrypted images, respectively, as shown in Fig. 5. With the periodic boundary treatment, the image encryption using $n$ iterations of the Arnold transform may be written as

$$
I(x, y)^{(k)}=I D(a, b)^{(k-1)}(\bmod N)
$$

where $k=1,2, \cdots, n$, and $I(x, y)^{(0)}=I(a, b)$. The Arnold transform matrix is given as $D$ in (7) and $I$ is an $N \times N$ image field.

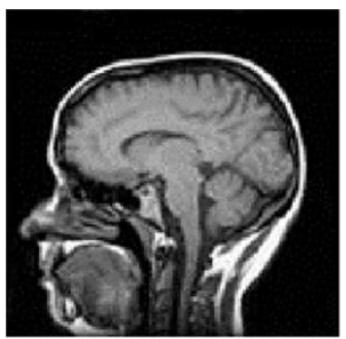

(a)

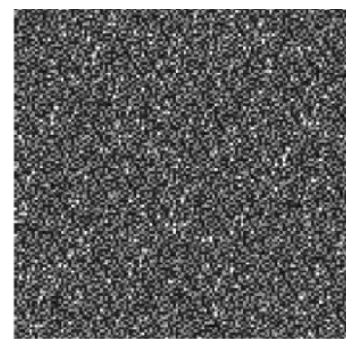

(b)
Fig.5. The 5 iterations of the Arnold transforms of a medical information image: (a) Medical information image. (b) Information image scrambled by the Arnold transforms.

The encrypted image may be inverted by applying the inverse of the Arnold matrix $D n$ times as follows:

$$
I(a, b)^{(k)}=I D^{-1}(x, y)^{(k-1)}(\bmod N)
$$

where $(x, y)^{(0)}$ is the pixel of the encrypted image. An original image may reappear after $T$ iterations, depending on the size of the given image. 
The periodicity $T$ depends on the size of the images, as shown in Table 1.

TABLE 1. PERIODICITY OF ARNOLD TRANSFORMS

\begin{tabular}{|l|c|c|c|c|}
\hline $\boldsymbol{N}$ & 128 & 256 & 480 & 512 \\
\hline $\boldsymbol{T}$ & 96 & 192 & 240 & 384 \\
\hline
\end{tabular}

\section{DATA Hiding Method}

\section{A. The embedding process}

The embedding process consists of two phases. First, the Arnold transform is applied on the host image following the wavelet transform. Second, to encrypt the information data, the Fresnelet transform is performed with a single FFT (fast Fourier transform) approach up to the first level by specifying the sampling interval and distance as key parameters. In this case, the sub-bands of information data are of complex data. In the immediate phase, we separate the complex data into real and imaginary parts, respectively.

In this way, we obtain the two sets of four sub-band images (approximation details, horizontal details, vertical details, and diagonal details). Applying the IDWT (inverse discrete wavelet transforms) on each set separately, we obtain two coded images. These two images of real and imaginary parts are embedded into the same size as the sub-bands of the host image after performing the DCT on each sub-band. Real part which contains important detail of information data is embedded into approximation and horizontal sub-bands of host image, whereas imaginary part is embedded into vertical and diagonal detail of host image. To obtain information embedded data, the IDCT is performed on the each embedded sub-bands of cover image. It is used for distributing the amplitude values of Fresnelet coefficients of information data image of the processed data as shown in Fig. 6.

In this process, the approximate and horizontal detailed parts are modified with real part data, which leads to enhance the strength of the information data, and the vertical and diagonal detailed parts are modified with imaginary part data to balance the transparency of the host image. The intensity of the transformed pattern is embedded into the host image with a strength factor. After embedding, the IDWT is performed to write out the image file as an embedded image for digital media handling or internet transmission.

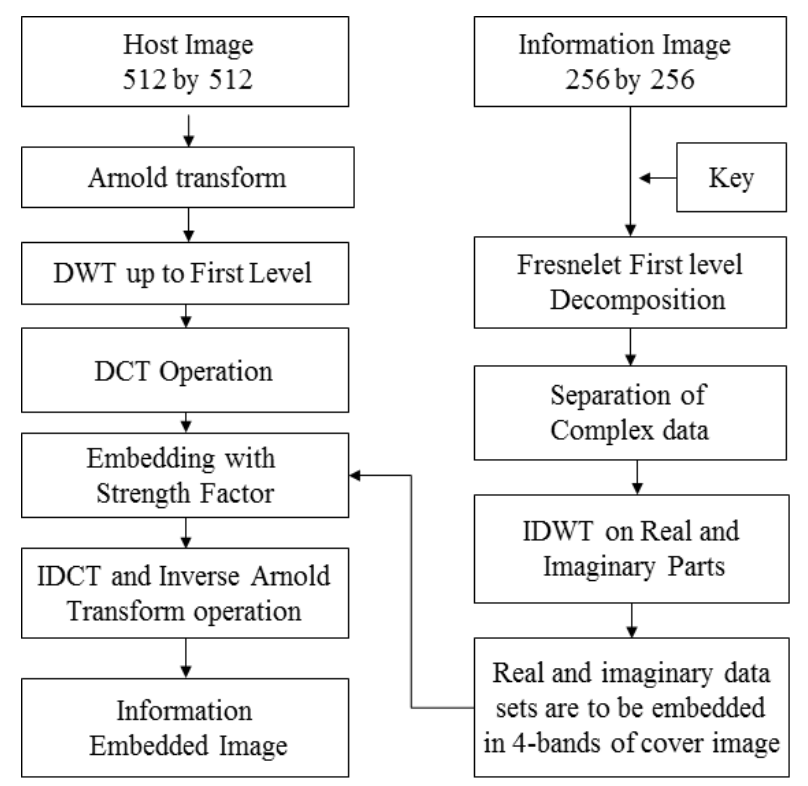

Fig.6. The schematic diagram of the embedding process.

\section{B. The extraction process}

In order to extract the embedded information data, the Arnold transform followed by DWT (discrete wavelet transform) is performed on the embedded data to obtain the sub-bands of the approximate data $\left(E_{a}\right)$, horizontal data $\left(E_{h}\right)$, vertical data $\left(E_{v}\right)$, and diagonal data $\left(E_{d}\right)$. The Arnold transform followed by DWT up to the first level is also performed on the original host image, and then each sub-band of the host date is subtracted from the sub-band data $E_{a}, E_{h}$, $E_{v}$, and $E_{d}$, respectively.

Applying DCT on the difference data is performed to extract the information coded data. After DWT is applied, the real and imaginary parts are separated to reconstruct the complex sub-band images for performing the inverse Fresnelet transform. As a result, the extraction information image is obtained in the form of complex. The whole process of the extraction is shown in Fig. 7.

\section{NumERICAL SimUlation AND Evaluation}

\section{A. Simulation and measurement of quality}

In this simulation, the size of all the cover images is $512 \times 512$ and the size of the information images is $256 \times$ 256. To analyze the algorithm, MATLAB simulations are performed separately for embedding and extraction of the information data image. 


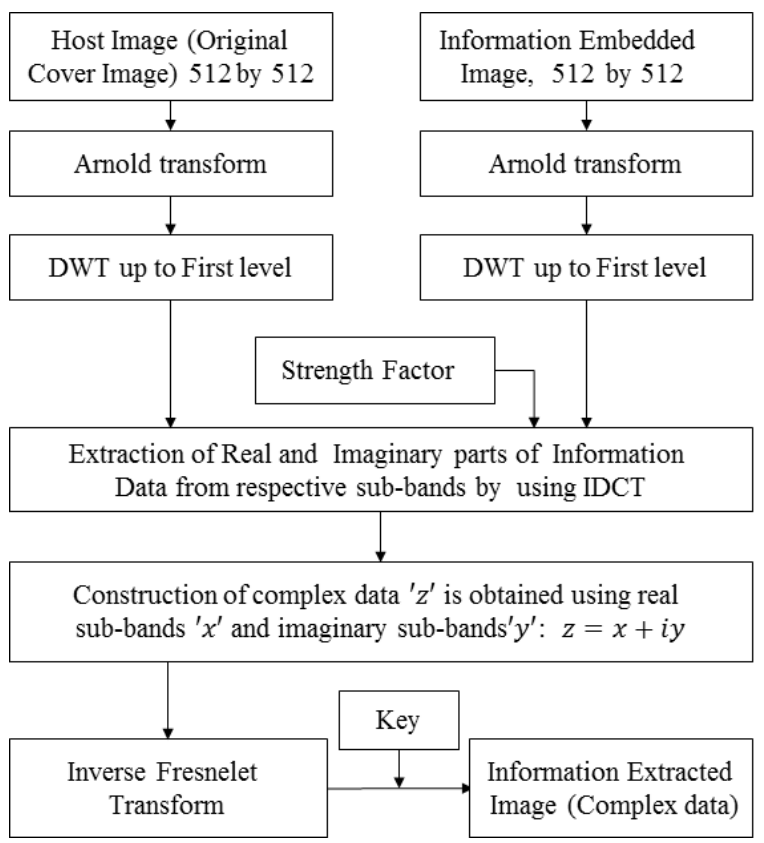

Fig.7. The schematic diagram of the extraction process.

The distance $z$ and the wavelength $\lambda$ are key parameters of the Fresnelet transform that improve the security level of the embedded images. In the proposed method, we considered a wavelength; $\lambda=632.8 \mathrm{~nm}$, sampling interval; $\Delta=10 \mathrm{~nm}$, and the distance; $d=200 \mathrm{~cm}$. The Arnold transform iteration may also be considered as an additional key parameter. The correlation coefficient (CC) is used to measure the quality of the extracted information data with respect to the original information data before embedding [31]. Specially, ultrasonic detection in medical images is based on amplitude for nondestructive evaluation [32].

In the correlation approach, each signal is correlated with its nearest neighbors for application of matched filtering. A confidence level specifies the relative degree of confidence between two images, with lower to higher values of 0 to 1 . The correlation coefficient is given by

$$
C C=\frac{\sum_{i} \sum_{j}\left(I_{e m b}(i, j)-\bar{I}_{e m b}\right)\left(I_{e x t}(i, j)-\bar{I}_{e x t}\right)}{\sqrt{\sum_{i} \sum_{j}\left(I_{e m b}(i, j)-\bar{I}_{e m b}\right)^{2}} \sqrt{\sum_{i} \sum_{j}\left(I_{e x t}(i, j)-\bar{I}_{e x t}\right)^{2}}}
$$

where $\bar{I}_{e m b}$ and $\bar{I}_{\text {ext }}$ are the averages of embedded and extracted information data images, respectively. In addition, the PSNR (peak signal noise ratio) is a common estimate of the quality of the embedded image data [33].

Higher values of PSNR indicate better imperceptibility of the embedded information data with respect to the cover data:

$$
P S N R=10 \log \left(\frac{255^{2}}{M S E}\right)
$$

with

$$
M S E=\frac{1}{m n} \sum_{i=0}^{m-1} \sum_{j=0}^{n-1}\left[I_{O}(i, j)-I_{E}(i, j)\right]^{2}
$$

where $M S E$ is the mean square error between original image $I_{O}$ and embedded image $I_{E}$. The difference $I_{O}-I_{E}$ is an encrypted information data that considered as a noisy like image as shown in Fig. 9-c and Fig. 9-f. Moreover, in order to evaluate the perception quality of human visual system, the structural similarity index measure (SSIM) is used to measure the distortion difference two images. The SSIM metric is designed by modeling any image distortion as a combination of three factors: loss of correlation, luminance and contrast distortions. The SSIM comparison for measuring the perceptual quality between two images is defined as

$$
\begin{gathered}
\operatorname{SSIM}(a, b)=l(a, b) c(a, b) s(a, b) \\
l(a, b)=\frac{2 \mu_{a} \mu_{b}+C_{1}}{\mu_{a}^{2}+\mu_{b}^{2}+C_{1}} \\
c(a, b)=\frac{2 \sigma_{a} \sigma_{b}+C_{2}}{\sigma_{a}^{2}+\sigma_{b}^{2}+C_{2}} \\
s(a, b)=\frac{2 \sigma_{a b}+C_{3}}{\sigma_{a b}+C_{3}}
\end{gathered}
$$

where $l(a, b)$ is the function of luminance comparison to measure the images closeness on the base of mean luminance $\mu_{a}$ and $\mu_{b}$ of 2D images $a$ and $b$. Maximum value of $l(a, b)$ is equal to 1 , if and only if $\mu_{a}=\mu_{b}$. The second term $c(a, b)$ in (13) is used to measure the contrast on the base of standard deviation $\sigma_{a}$ and $\sigma_{b}$.

The maximal value of contrast term as given in (15) is achieved when $\sigma_{a}=\sigma_{b}$.The third term $s(a, b)$ in (13) measures the structure comparison between the two images. In (16), $\sigma_{a b}$ is the covariance that is useful to analyze the correlation between two images. In the equations (14)-(16) above, the positive constants $C_{1}, C_{2}$, and $C_{3}$ are used to avoid the null denominator. The quality value of SSIM is varied in $[0,1]$. The value of 1 shows that two images are having the same quality and the value of 0 shows no correlation between the two image.

\section{B. Evaluation criteria}

In this algorithm, the information image data is embedded in a cover image to increase the security level and improve the imperceptibility of the cover media. For evaluation of the proposed method, the PSNR and SSIM of cover image after embedding of information data are evaluated as shown in Table. 2. Also the CC factor and SSIM of the information image after extraction are analyzed as shown in Table. 3 . The numeric results show the superior performance of the proposed algorithm.

A slight effect may be presented due to auto focus nature of the Fresnelet transform, where the Fresnelet sparsity criterion is employed for extraction of information data in the form of complex values, as shown in Fig. 8 through 11. The corresponding PSNR and SSIM are shown in Tables 2 for signature image 1 (the brain image in Fig. 8-a), signature image 2 (the backbone image in Fig. 8-b), and signature image 3 (the USAF target image in Fig. 8-c). Note that the signature images are high resolution information image data. 


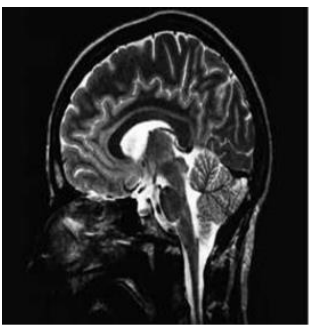

(a)

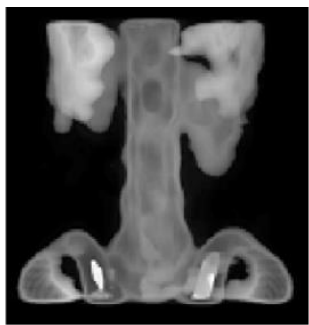

(b)

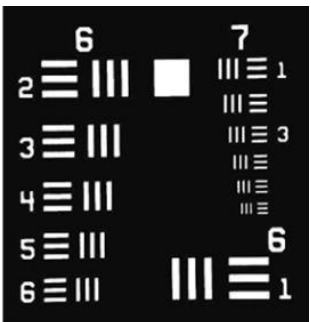

(c)

Fig.8. High resolution signature image data with size $256 \times 256$ : (a) the brain medical image, (b) the backbone medical image, and (c) the USAF test image.

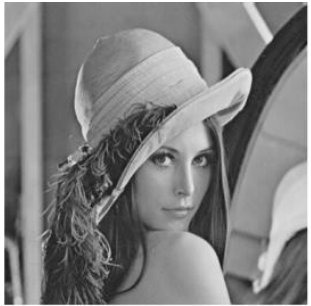

(a)

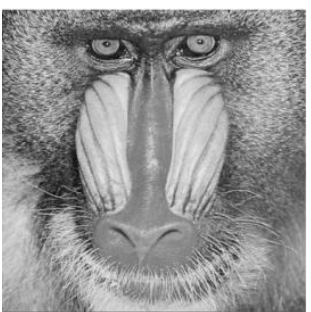

(d)

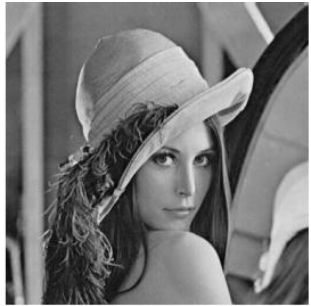

(b)

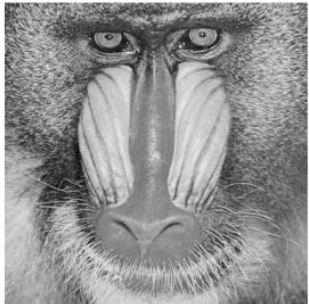

(e)

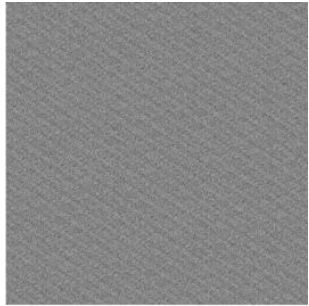

(c)

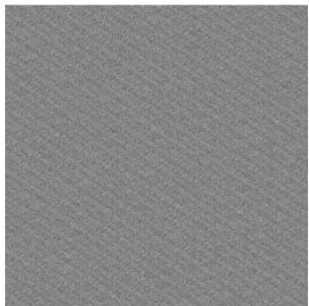

(f)

Fig.9. The cover images embedded with signature image 1 as shown in Fig. 8-a: (a) the original cover Lena image, (b) the cover Lena image embedded with signature image 1 (c) the difference between the original cover Lena image and the embedded Lena image with signature image 1 where MSE is 4.3273, (d) the original cover Mandrill image, (e) the embedded Mandrill image with signature image 1, and (f) the difference between the original cover Mandrill image and embedded Mandrill image with signature image 1 where MSE is 4.3268.

Table 2. PSNR and SSIM of the embedded images: Lena and Mandrill

\begin{tabular}{|c|c|c|c|c|}
\hline \multirow{2}{*}{ Test data } & \multicolumn{2}{|c|}{$\begin{array}{c}\text { Extraction from } \\
\text { embedded Lena image }\end{array}$} & \multicolumn{2}{c|}{$\begin{array}{c}\text { Extraction from } \\
\text { embedded Mandrill image }\end{array}$} \\
\cline { 2 - 5 } & $\boldsymbol{P S N R}$ & SSIM & PSNR & SSIM \\
\hline Signature image 1 & 38.8483 & 0.9982 & 40.8311 & 0.9985 \\
\hline Signature image 2 & 36.0625 & 0.9966 & 36.0638 & 0.9956 \\
\hline Signature image 3 & 36.7419 & 0.9971 & 38.7388 & 0.9976 \\
\hline
\end{tabular}




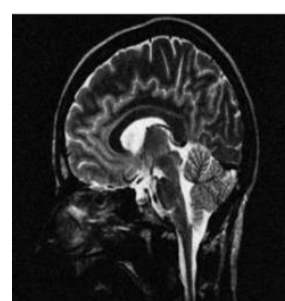

(a)

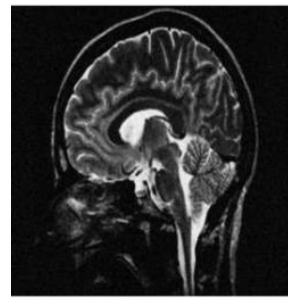

(d)

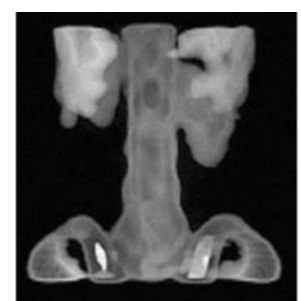

(b)

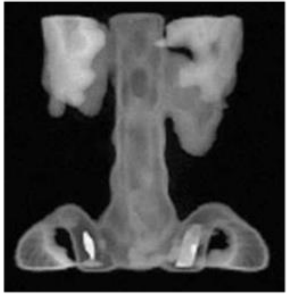

(e)

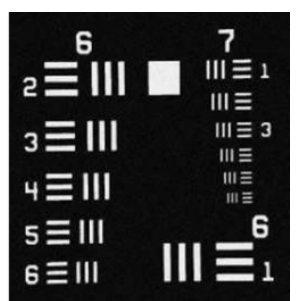

(c)

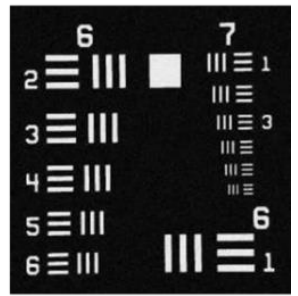

(f)

Fig.10. Extraction of signature images from embedded images of Lena and Mandrill: (a) the signature image 1 extracted from embedded Lena image, (b) the signature image 2 extracted from embedded Lena image, (c) the signature image 3 extracted from embedded Lena image, (d) the signature image 1 extracted from embedded Mandrill image, (e) the signature image 2 extracted from embedded Mandrill image, and (f) the signature image 3 extracted from embedded Mandrill image.

Table 3. CC factor and SSIM of signature data extracted from embedded images: Lena and Mandrill

\begin{tabular}{|c|c|c|c|c|}
\hline \multirow{2}{*}{ Test data } & \multicolumn{2}{|c|}{$\begin{array}{c}\text { Extraction from } \\
\text { embedded Lena image }\end{array}$} & \multicolumn{2}{c|}{$\begin{array}{c}\text { Extraction from } \\
\text { embedded Mandrill image }\end{array}$} \\
\cline { 2 - 5 } & CC factor & SSIM & CC factor & SSIM \\
\hline Signature Image 1 & 0.9965 & 1.0000 & 0.9947 & 1.0000 \\
\hline Signature Image 2 & 0.9978 & 1.0000 & 0.9977 & 1.0000 \\
\hline Signature Image 3 & 0.9981 & 1.0000 & 0.9972 & 1.0000 \\
\hline
\end{tabular}

Table 4. The comparison of image quality using PSNR (dB) and embedding capacity (bits)

\begin{tabular}{|c|c|c|c|c|}
\hline \multirow{2}{*}{$\begin{array}{c}\text { Cover Images } \\
\text { Measurements }\end{array}$} & \multicolumn{2}{|c|}{ Lena $(512 \times 512)$} & \multicolumn{2}{|c|}{ Baboon $(512 \times 512)$} \\
\hline & Capacity (bits) & $\operatorname{PSNR}(d B)$ & Capacity (bits) & $\operatorname{PSNR}(d B)$ \\
\hline Celik et al.'s [18] & 74600 & 38.00 & 15176 & 38.00 \\
\hline Xuan et al.'s [19] & 85507 & 36.60 & 14916 & 32.80 \\
\hline Tian et al.’s [20] & 233067 & 29.97 & 95852 & 29.41 \\
\hline Alattar et al.'s [21] & 173655 & 36.60 & 86264 & 36.60 \\
\hline Wu-Tsai's al.'s [26] & 51219 & 38.94 & 57146 & 33.43 \\
\hline Kamstra et al.'s [22] & 135547 & 35.20 & 103653 & 30.12 \\
\hline Chin-C. C et al.'s [32] & 36850 & 30.34 & 35402 & 26.46 \\
\hline Luo, W et al.'s [27] & 66064 & 38.80 & 68007 & 33.33 \\
\hline Mandal et al.'s [24] & 216000 & 40.92 & 216000 & 40.97 \\
\hline Proposed Method & 263222 & 38.65 & 263222 & 38.65 \\
\hline
\end{tabular}

The SSIM and CC factor evaluations of Table. 4 show the competency of proposed method. However, it is not appropriate to compare the extraction results of information data with those of other existing methods due to complex values extraction results of proposed method (usually, existing methods do not extract the secret image in the form of complex values). To further prove our scheme's performance on hiding capacity, we compare our proposed scheme with other existing schemes. That is, in order to compare the quality of the embedded images and view the degradation caused by embedding a hidden data, it is necessary to look at embedded cover images with a special payload size [34]. Usually, the resulting perceived quality or PSNR and payload or bits per pixel (bpp) are the two most commonly used criteria for evaluating the performance of reversible data hiding techniques [35]. 
Table. 4 shows the comparison of our results with Celik et al.'s [36] technique based on compressing the quantization residuals of pixels instead of compressing LSB planes to obtain more extra space for embedding secret data. The compressed residuals and secret data are concatenated and embedded into the cover image. Xuan et al.'s [37] proposed a scheme based on integer wavelet transform (IWT) and created more space in high frequency sub-bands. Tian et al.'s [38] proposed a high capacity reversible data embedding technique that is called difference-expansion (DE) embedding. Alattar et al.'s [39] extended Tian's scheme by introducing the differences expansion of a vector to obtain more extra space for embedding secret data. Afterwards, many techniques, such as Kamstra et al.'s algorithm [40], Chin-C et al.'s [41], Luo et al.'s [42], and Mandal et al.'s [43] have been proposed to increase the embedding capacity and keep the distortion low. But all such methods do not maintained the high resolution images (e.g., medical images etc.) in their extraction phase. However, it is found that hiding capacity of Mandal et al.'s method is higher than other such prevailing techniques, but its hiding capacity is less than our proposed method with cover image size $512 \times 512$ and payload size $256 \times 256$. Moreover, overall performance of our proposed method shows significant improvement compared with other existing schemes under typical circumstances as demonstrated in Table. 4. Besides this, our proposed method is highly capable to preserve the high resolution of information data images at their extraction stage. Additionally, multi-key establishment for extraction phase provides the high end confidentiality to concealed information data, whereas appropriate encryption develops the great imperceptibility for embedded image. The experimental results presented in Table 4 for comparison of image quality using PSNR (dB) and embedding capacity (bits) are referred to their original experimental results in their papers. The size of payload data (bits) in a cover image can be modified without deteriorating the integrity of the cover image [35]. However, it is not possible to simultaneously maximize robustness, imperceptiveness, and capacity. Therefore, the acceptable balance of these items must be dictated by the application. For example, an information-hiding scheme may forgo robustness in favor of capacity and low perceptibility, whereas a watermarking scheme, which may not require large capacity or low perceptibility, would certainly support increased robustness [6].

\section{CONCLUSION}

The present paper proposes a novel data hiding scheme to increase the security and privacy of medical information image data by integrating both steganography and cryptography, based on the Fresnelet transform. The main feature of the proposed method is a multi-scale distribution of information on the Fresnelet transform domain with robust key parameters for security, so that the very small distortion in the cover image provides sufficient privacy for the embedded information.

In particular, the Fresnelet decomposition provides robustness to leakage of the energy of information data during the embedding process. For greater security, a combination of the Arnold transform and DCT has been employed in the embedding process. Numerical simulation results have been analyzed with PSNR, SSIM, MSE, and CC factors. Higher values of PSNR, SSIM, and CC factors and smaller value of MSE show the effectiveness of the proposed novel algorithm to achieve great imperceptibility of embedded information image data with required security.

\section{REFERENCES}

[1] Munchh, H., Engelmann, U., Schroeter, A., and Meinzer, H.: 'Webbased distribution of radiological images from PACS to EPR', International Congress Series., 2003, 1256, pp. 873-879.

[2] Boucherkha. S and Benmohamed . M.: 'A Lossless Watermarking Based Authentication System For Medical Images'. Proc. of International Conf on Computational Intelligence, Istanbul, Turkey, 2004, pp. 240-243.

[3] Pitt, , et al.: 'Imaging cortical lesions in multiple sclerosis with ultrahigh-field magnetic resonance imaging',Archives of Neurology, 67(7), 2010, pp. 812-818.

[4] Al-Frajat A. K. and Jalab H..: 'On the differences between hiding information and cryptography techniques: An overview', Journal of Applied Sciences, 2010, vol. 10, (15), pp. 1650-1655.

[5] Dickman, S. D.: 'An Overview of Steganography', JMU-INFOSEC-TR2007-002, doi=10.1.1.137.5129

[6] Li, J. He, J. Huang and Y. Q. Shi.: 'A Survey on Image Steganography and Steganalysis', Journal of Information Hiding and Multimedia Signal Processing, 2(2), 2011, pp. 142-172.

[7] Wu, H.-C et al.: 'Image steganographic scheme based on pixel-value differencing and LSB replacement methods'. Vision, Image and Signal Processing, IEE Proceedings, 152(5), 2005, pp. 611-615.

[8] Luo, W et al.: 'Edge Adaptive Image Steganography Based on LSB Matching Revisited' IEEE Transactions on Information Forensics and Security, 5(2), 2010, pp. 201-214.

[9] Fridrich, J., Goljan, M., Du, and R .: 'Detecting LSB steganography in color, and gray-scale images', IEEE Multimedia, 8(4), 2001, pp. 22-28.

[10] Dumitrescu, S., Wu,X. and Wang, Z.: 'Detection of LSB steganography via sample pair analysis," IEEE Trans. Signal Process., 51(7), 2003, pp. 1995-2007.

[11] Yang, H., et al.: 'A High-Capacity Image Data Hiding Scheme Using Adaptive LSB Substitution', Journal of Radioengineering,18(4), 2009, pp. 509-516

[12] Ker, A. D.: 'A fusion of maximum likelihood and structural steganalysis', in Proc. 9th Int. Workshop on Information Hiding, 2007, vol. 4567, pp. 204-219.

[13] Zeng, X.-T et al.: 'A Reversible Data Hiding Scheme using Center Pixel Difference', Journal of Multimedia. 5(4), 2010, pp. 377-384

[14] Chang, C.-C., Lin, C.-C., Tseng, C.-S., \& Tai, W.-L.:“ Reversible hiding in DCT-based compressed images', Information Sciences, 177(13), 2007, 2768-2786.

[15] Iwata, M., Miyake, K., and Shiozaki, A.: 'Digital Steganography Utilizing Features of JPEG Images, IEICE Trans. Fundamentals, E87A(4), 2004, pp. 929-936.

[16] Lin , C. Y., Chang, C. C., and Wang, Y. Z.: 'Reversible Steganographic Method with High Payload for JPEG Images', IEICE Trans. Information and Systems, 91-D(3), 2008, pp. 836-845.

[17] Lin, C. C. and Shiu, P. F.: 'DCT-based reversible data hiding scheme, in Proc. of the 3rd International Conference on Ubiquitous Information Management and Communication (ICUIMC'09), 2009, pp. 327-335.

[18] Conway, M.: 'Code Wars: Steganography, Signals Intelligence, and Terrorism', Knowledge Technology \& Policy, Springer, 16(2), 2003, pp. 45-62.

[19] Song, Set al .: 'A Novel Secure Communication Protocol Combining Steganography and Cryptography', Elsevier Inc, Advanced in Control Engineering and Information Science, 15, 2011, pp. 2767 - 2772.

[20] Tso, H.-K. and Lou, D.-C.: 'Medical image protection using secret sharing scheme', in Proc. of International Conference on Ubiquitous Information Management and Communication, 2012, pp. 93-93.

[21] Shamir, A.: 'How to share a secret', Communication of the ACM, 22(11), 1979, pp. 612-613. 
[22] Fallahpour, M., Megias, D., \& Ghanbari, M. .: 'Reversible and highcapacity data hiding in medical images', IET Image Processing, 5(2), 2011, pp. 190-197.

[23] Liebling, M., Blu,T., and Unser, M.: 'Fresnelets: New Multiresolution Wavelet Bases for Digital Holography', IEEE Transactions on Image Processing, 2003, vol. 12,(1), pp. 29-43.

[24] Yang, J.: 'Algorithm of image information hiding based on new antiArnold transform and Blending in DCT domain'. Proc. of IEEE Conf on Communication Technology, Nanjing, China, 2010, pp. $312-315$.

[25] Sun, M. and Zhu, Z.: 'Digital Image Hiding Technology Based on Three-Dimension Object Digital Holography Encoded by DoubleRandom Phase," 2010. '. Proc. of Photonics and Optoelectronic, 2010, pp. 1-4.

[26] Nazeer, M., and Kim, D. G.: 'A novel Fresnlet based robust data hiding algorithm for medical images'. Proc.of IEEE Conf on Imaging Systems and Techniques, Manchester, U.K, 2012, pp. 213-216.

[27] Liebling, M., \& Unser, M.: 'Autofocus for Digital Fresnel Holograms by Use of a Fresnelet-Sparsity Criterion', Journal of the Optical Society of America, 21(12), 2004, pp. 2424-2430.

[28] Greenspan, H et al.:'Super-Resolution in Medical Imaging', The Computer Journal, 52(1), 2009, pp. 43-63.

[29] Kang, S., and Aoki, Y.: 'A multiple data embedding technique for DRM using Fresnel transform'. Proc.of IEEE Conf on Electrical and Computer Engineering, Saskatoon, Canada, 2005, pp. 2174-2177.

[30] Dandapat, S., Xu, J., Chutatape, O., and Krishnan, S.: 'Wavelet transform domain data embedding in a medical image'. Proc. of IEEE Conf on Engineering in Medicine and Biology Society, San Francisco, CA, 2004, pp. $1541-1544$.

[31] Unfeng, Li and Wenzhan, Dai.: 'Image quality assessment based on the correlation coefficient and the 2-D discrete wavelet transform'. Proc. of IEEE Conf on Automation and Logistics, 2009, pp. 789 -793.

[32] Cepel, R et al.: 'Spatial Correlation Coefficient Images for Ultrasonic Detection' IEEE Transactions on Ultrasonics, Ferroelectrics and Frequency Control, 54(9), 2007, pp. 1841-1850.

[33] Wang, $Z$ et al.: 'Image quality assessment: from error visibility to structural similarity',IEEE Transactions on Image Processing, 13(4), 2004, pp. 600-612.

[34] Chen, W.-J et al.: 'High payload steganography mechanism using hybrid edge detector', Expert Systems with Applications. 37(4), 2010, pp. 3292-3301.

[35] Mare, S. F et al.: 'High capacity steganographic algorithm based on payload adaptation and optimization'. Proc. of IEEE Conf on Applied Computational Intelligence and Informatics, 2012, pp. 87-92.

[36] Celik, M.U, et al. : 'Lossless watermarking for image authentication: A new framework and an implementation," IEEE Transactions on Image Processing, 15,(4), 2006, pp.1042-1049.

[37] Xuan, $G$ et al.: 'Algorithm of image information hiding based on new anti-Arnold transform and Blending in DCT domain', IEE Electron. Lett., vol. 38, (25), 2002, pp.1646-1648.

[38] Tian, J.: 'Reversible data embedding using a difference expansion', IEEE Trans. Circuits and Systems for VideoTechnology, 13(8), 2003, pp.890-896.
[39] Alattar, A.M.: 'Reversible watermark using the difference expansion of a generalized integer transform', IEEE Trans. Image Processing. 13(8), 2004, pp.1147-1156.

[40] Kamstra, L., and Heijmans, H.: 'Reversible data embedding into images using wavelet techniques and sorting', Image Processing, IEEE Transactions on, 14(12), 2005, 2082-2090.

[41] Chen, W.-J et al.: 'High payload steganography mechanism using hybrid edge detector', Expert Systems with Applications. 37(4), 2010, pp. 3292-3301.

[42] Luo, W et al.: 'Edge Adaptive Image Steganography Based on LSB Matching Revisited' IEEE Transactions on Information Forensics and Security, 5(2), 2010, pp. 201-214.

[43] Mandal, J. K, et al.: 'A Novel Genetic Algorithm Based Data Embedding Technique in Frequency Domain Using Z Transform', Proc. of Advances in Intelligent S.

\section{AUTHORS PROFILES}

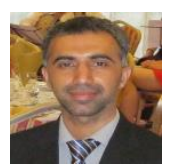

The first author of this paper, Mr. Nazeer Muhammad is currently pursuing the Ph.D. degree in the department of Applied Mathematics, Hanyang University, South Korea. In 2010, he received the prestigious Pakistan Government higher education commission (HEC) scholarship award. His interests are digital signal watermarking, data hiding, digital image denoising, digital holography, OFDM, and information theory.

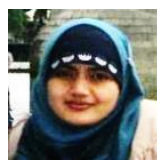

Ms. Nargis Bibi is a postgraduate research student in the School of Computer Science, University of Manchester. She received her M.Sc from Fatima Jinnah Women University (FJWU), Pakistan. She is currently employed in FJWU as lecturer. Her interests are digital signal processing, OFDM, coding theory and information theory.

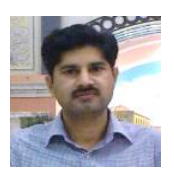

Mr. Yasir Mehmood Malik is a Specialist registrar at Rashid Hospital, Dubai in Department of Neourology. He received his Bachelor of Medicine and Bachelor of Surgery (MBBS) from King Edward Medical College, Lahore, Pakistan. He has completed his course for Fellow of College of Physicians and Surgeons (FCPS), Pakistan in 2011. His

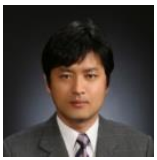
area of interest is interventional Neurology.

The corresponding author of this paper, Kim, DaiGyoung* is a professor of the Department of Applied Mathematics, Hanyang University. He received a B.S. (1983) and M.S (1986) in Mathematics from Hanyang University, and a Ph.D. (1994) in Applied Mathematics from Purdue University. Before joining the Hanyang University faculty in 1995, Professor Kim had been on the IMA of University of Minnesota as a postdoctoral fellow. Also, he was a visiting scholar of IMAGERS UCLA Image Processing Research Group during 2003 2004.

Professor Kim has been teaching various courses of applied mathematics such as linear algebra, numerical analysis, mathematical modeling, real analysis, functional analysis, numerical PDE, wavelet theory and applications. His research mainly focuses on the numerical PDE applied to signal/image processing, wavelet theory and its applications, and 3D holography as well as scientific computations in physics and chemistry.

Readers can contact the author at dgkim@hanyang.ac.kr. 